\title{
Recursos virtuales para trabajar la probabilidad en la enseñanza de matemáticas en Educación Primaria
}

\section{Virtual resources for teaching probability in mathematics of Primary \\ Education}

\author{
José Miguel Contreras García ${ }^{1}$, Karen Ruiz ${ }^{2}$, Felipe Ruz Ángel ${ }^{3}$, Elena Molina-Portillo ${ }^{4}$ \\ 12Departamento de Didáctica de la Matemática, Universidad de Granada, España (jmcontreras@ugr.es) \\ ${ }^{2}$ Departamento de Didáctica de la Matemática, Universidad de Granada, España (karenruizreyes@ gmail.com) \\ ${ }^{3}$ Departamento de Didáctica de la Matemática, Universidad de Granada, España (felipe.ruz.angel@ gmail.com) \\ ${ }^{4}$ Departamento de Didáctica de la Matemática, Universidad de Granada, España (elemo@ugr.es)
}

Recibido el 19 de septiembre de 2018; revisado el 25 de septiembre de 2019; aceptado el 15 de marzo de 2019; publicado el 1 de junio de 2019

\section{RESUMEN:}

El interés mostrado en los últimos años por aunar la enseñanza de las matemáticas y las tecnologías de la información y comunicación (TIC) por parte de los organismos oficiales nos ha llevado a plantearnos la necesidad de evaluar los recursos virtuales disponibles para la enseñanza de la probabilidad en educación primaria. La enseñanza de la probabilidad, dado su carácter experimental, especialmente en edades tempranas, necesita de metodologías basadas en el uso de recursos que muestren el sentido de la aleatoriedad y el cálculo de probabilidades. Estas herramientas, profundizan en el significado de los conceptos, muestran el comportamiento de los procedimientos y propiedades y ayudan a los estudiantes a superar sus dificultades, principalmente en la identificación de los sesgos probabilísticos. En este trabajo proponemos un catálogo de recursos útiles, convenientemente analizados a partir de los elementos del enfoque ontosemiótico de la instrucción matemática, que pueden ser utilizados como complemento a la enseñanza formal del tema de probabilidad.

PALABRAS CLAVE: EDUCACIÓN PRIMARIA, RECURSOS DE INTERNET, ENSEÑANZA DE LA PROBABILIDAD.

\section{ABSTRACT:}

In the last years, educational community has shown interest for introduce the information and communication technologies (ICT) in the teaching of mathematics. This produce the need to evaluate the available virtual resources for teaching probability in Primary Education. Due to the experimental nature of the teaching of probability, especially that produced in early ages, it is needed methodologies based on the use of resources that shows the random sense and use of probability. These virtual tools delve into the concepts' meanings, show procedures and properties relevance and help to get over the difficulties mainly related to probabilistic biases. From Ontosemiotic Approach of Mathematical Instruction, this paper propose some of the most useful virtual tools which can be used in the formal teaching of probability
KEYWORDS:
PROBABILITY,
PRIMARY, INTERNET RESOURCES, TEACHING 


\section{INTRODUCCIÓN}

En las últimas décadas, la probabilidad ha alcanzado un papel relevante en los diferentes currículos nacionales de primaria, principalmente por ser una herramienta que permite al alumno interpretar concepciones de la vida diaria (Batanero, Burril, y Reading, 2011; Burril, 2006; Jones, Langrall, y Mooney, 2007; Gómez y Contreras, 2013, 2014). Otro aspecto en el que los diferentes currículos hacen hincapié es utilizar las TIC para facilitar la búsqueda de ejemplos y el análisis de estos por parte del alumnado (MEC, 2014; Consejería de Educación, 2015).

Como indica Galmacci (2001), la probabilidad es una de las materias que ha tenido mayor influencia de la tecnología y en particular de Internet. Es por ello que se presenta la necesidad de confluir la probabilidad y las TIC para facilitar el aprendizaje de las concepciones probabilísticas en nuestros estudiantes.

En este trabajo presentamos un conjunto de recursos virtuales o applet, útiles para la enseñanza de la probabilidad en educación primaria, que han sido analizados y clasificados utilizando herramientas del enfoque ontosemiótico desarrolladas por Godino y colaboradores. Para la selección de estos recursos se han seguido los criterios de selección y evaluación de Godino, Contreras y Font (2006) y Contreras $(2009,2011)$.

\section{INTERÉS DIDÁCTICO DE LOS RECURSOS VIRTUALES}

Los modelos sobre el conocimiento profesional del profesor de matemáticas sugieren que los conocimientos probabilísticos en sí mismos no son suficientes para que los docentes puedan enseñar probabilidad de una manera efectiva y desarrollar en sus estudiantes un adecuado razonamiento probabilístico.

Son muchos los autores que defienden el interés de utilizar recursos virtuales en el aula de matemáticas, pues permiten plantear situaciones motivadoras, refuerzan el interés y la metacognición de los estudiantes y les hace descubrir conexiones con la vida cotidiana. Como indican Godino et al. (2006), los recursos didácticos virtuales pueden ser el soporte para el planteamiento de problemas y situaciones didácticas que promuevan la actividad y reflexión matemática.

El uso de recursos apoya una pedagogía constructivista, promoviendo un aprendizaje profundo, partiendo de las creencias previas que los estudiantes pueden revisar al resolverlas. A la vez, el análisis y discusión de las soluciones a las mismas exige al alumnado una reflexión sobre sus propios procesos de pensamientos, lo que es tan importante como el aprendizaje de la solución correcta y un paso vital para alcanzar la capacidad matemática abstracta (Falk y Konold, 1992).

Otro aspecto a tener en cuenta es el interés de distintas instituciones, como, por ejemplo, la International Association for Statistics Education (IASE) por la influencia de las TIC en la probabilidad y su enseñanza, ya que ofrecen un abanico de herramientas con un potencial muy importante para contribuir a la labor docente (Nóbile y Luna, 2015).

En particular, por los cambios que deben llevarse a cabo en el contenido y la metodología, tanto en el aprendizaje como en las actitudes de los estudiantes. En este sentido, podemos encontrar distintos autores que hacen referencia a estos elementos. Por ejemplo, Mills (2004) recomienda el uso de métodos de simulación para la enseñanza de conceptos probabilísticos, ya que proporcionan una mayor comprensión de conceptos abstractos por parte de los estudiantes. Sada (2011) menciona las ventajas del uso de applets a través de la utilización del ordenador en el aula, ya que facilita la comprensión, la representación e interpretación, la observación colectiva de resultados de un número elevado de simulaciones, favorece la dinámica del debate y el ritmo del aula. Barragués y Guisasola (2007) indican que los recursos virtuales toman un papel activo como herramienta para la construcción de modelos probabilísticos, tanto como herramienta de resolución de problemas, para profundizar en el significado de los conceptos, y como ayuda a los estudiantes para superar sus dificultades. Pérez et al. (2012) concluyen que los applets desarrollados son valiosos para ejercer la práctica docente, utilizándose como herramienta para fortalecer el proceso de enseñanza-aprendizaje. Visión que convive con la de Merriam, Caffarella y Baumgartner (2006), que señalan que la educación basada en los recursos disponibles en Internet contribuye a expandir el aprendizaje, perspectiva de la teoría del Conectivismo.

Osorio, Suárez y Uribe (2013) proponen el uso de recursos como complemento para mejorar la adquisición de los conceptos, como estrategia efectiva para fomentar el aprendizaje autónomo por parte del estudiante y de estímulo por los temas de probabilidad. 
En resumen, el interés de su uso recae en la mejora respecto a la enseñanza tradicional. Investigaciones como la de Herrera y Rodríguez (2011) señalan que la puesta en práctica de estas innovaciones revela resultados positivos respecto a períodos anteriores de docencia sin la utilización de esta clase de recursos.

De acuerdo a Godino, Font y Wilhelmi (2008) una tarea clave para el profesorado es valorar su práctica docente con la finalidad de favorecer el aprendizaje de los estudiantes. Para facilitar esta valoración, los autores describen diversos niveles de análisis que pueden aplicarse al estudio de los recursos didácticos virtuales: los sistemas de prácticas y objetos matemáticos implícitos en el trabajo con recursos, que según estos autores, permite identificar las configuraciones y objetos latentes en el trabajo de los estudiantes al resolver problemas con los recursos analizados; los procesos matemáticos requeridos y posibles conflictos semióticos de los estudiantes en el uso de recursos y las variantes del mismo encontradas en otros sitios de Internet.

Otro elemento del Enfoque Ontosemiótico es el concepto de idoneidad didáctica (Godino, Contreras y Font, 2006; Godino, Wilhelmi y Bencomo, 2005). En el caso de los recursos virtuales, Contreras (2011) indica que los recursos virtuales presentan un alto grado de idoneidad emocional ya que, sin duda, los recursos intrigan e interesan a la vez que muestran los elementos probabilísticos que la componen.

Podemos encontrar diferentes investigaciones que hacen uso del análisis semiótico de recursos virtuales, como las de Contreras (2009, 2011), Batanero, Fernándes y Contreras (2009), Contreras, Díaz, Arteaga, Gonzato y Cañadas (2011) o la de Ruiz (2013) que presentan un análisis de los objetos y procesos matemáticos implícitos en algunas soluciones correctas posibles de recursos útiles para la enseñanza de la probabilidad condicional. Estos trabajos explican distintos conflictos semióticos relacionados con los razonamientos erróneos más frecuentes en su solución y se señalan algunas implicaciones del uso de este problema en la enseñanza y formación de profesores.

\section{SELECCIÓN DE RECURSOS}

Para la localización de los recursos se ha realizado diferentes criterios de selección:

a) Exploración de los servidores de educación matemática que incluyen listados de recursos en Internet para la enseñanza de la probabilidad. b) Revisión del servidor de "Biblioteca virtual de recursos manipulativos" http://nlvm.usu.edu/es/nav/vlibrary.html.

c) Revisión de repositorios de recursos.

d) Revisión de servidores de recursos de organismos oficiales: NCTM (www.nctm.org), Proyecto Descartes del Ministerio de Educación (descartes.cnice.mec.es), etc.

e) Búsqueda directa en buscadores de Internet, utilizando palabras claves como "applet" más alguna de las siguientes "probabilidad", "educación primaria", "probability", etc.

Una vez localizado un recurso, se clasifica según su pertenencia a las diferentes categorías descritas por Contreras (2009, 2011):

1. Juegos.

2. Exploración de conceptos, con las subcategorías: 2.1) Azar, 2.2) Tipos de sucesos, 2.3) Experimentos aleatorios, 2.4) Simulaciones probabilísticas.

3. Problemas.

4. Lecciones o libros virtuales.

5. Vídeo-tutoriales.

\subsection{Juegos para la enseñanza de la probabilidad}

En esta categoría se incluyen diversos juegos encontrados en Internet cuya utilidad para la enseñanza es que hacen aflorar los errores y las concepciones erróneas de los estudiantes. En la formación de profesores, estos juegos permiten contextualizar la reflexión epistemológica sobre la probabilidad y otras ideas estocásticas fundamentales, así como analizar las posibles dificultades y obstáculos del alumnado (Godino, Batanero y Flores, 1999).

Ejemplo 1. En primer lugar, analizamos un recurso para la enseñanza de la probabilidad simple que permite explorar varios experimentos de extracción, sin reposición, de bolas de una caja y calcular la probabilidad de sacar una bola azul.

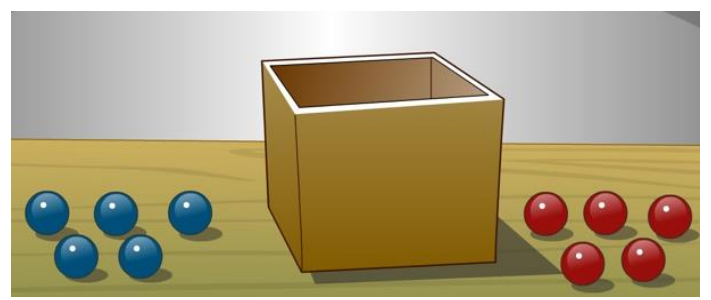

Figura 1. Experimento extraer una bola de una caja

http://repositorio.educa.jccm.es/portal/odes/matematicas/a zar_y_probabilidad/mt11_oa03_es/index.html 
El recurso muestra una caja y diez bolas (5 rojas y 5 azules) que son introducidas dentro de la caja. Posteriormente, el recurso pregunta: ¿cuál es la probabilidad de que saque Andrés, sin mirar, una bola de color azul? A continuación, el recurso explica el concepto de probabilidad simple, mediante la regla de Laplace, como cociente entre número de casos favorables y número de casos posibles. En una segunda fase, el recurso realiza cuestiones para que el usuario calcule la probabilidad de extraer la bola de un color, indicando los casos favorables y los posibles, de la bola que más probabilidad tenga de ser sacada de la caja, e indique qué color es más probable, mostrándole al usuario si es correcta o no la respuesta.

Una de las principales dificultades que pueden encontrar los estudiantes con este tipo de recursos es la interpretación del lenguaje del applet. Por ejemplo, el recurso explica cómo calcular probabilidades simples a partir de un muestreo sin reposición, pero siempre para el suceso "sacar una bola de color azul". Sin embargo, al finalizar, el recurso muestra una serie de problemas para el cálculo de probabilidades simples, donde se pide elegir "cual tiene más probabilidades de sacar una bola de color, rojo o azul" y rellenar la fórmula del Laplace. Por ello vemos que no se especifica si el suceso, al que se ha de realizar la probabilidad, es para un cierto color, como se mostraba en el ejemplo, y no se indica en ningún momento que solo se ha de rellenar la fórmula para el suceso con mayor probabilidad.

Respecto a la idoneidad didáctica, estos recursos presentan alta idoneidad matemática en el aprendizaje de los conceptos: experimento aleatorio, muestreo sin reposición, probabilidad, Regla de Laplace, etc. Las soluciones formales tendrán mayor idoneidad si se complementan con una solución intuitiva de las situaciones-problemas planteados en el recurso:

- Idoneidad cognitiva ya que presentan un lenguaje verbal que se utilizan términos de fácil comprensión y se complementan con una serie de representaciones pictóricas que guían al estudiante durante la exploración del recurso.

- Idoneidad interaccional, aunque esta dependerá de cómo organice el docente el trabajo en el aula. Será importante que los estudiantes trabajen en pareja o en grupos para que surja el conflicto y se expliciten las dudas que aparecen en el proceso. Será importante también organizar una puesta en común de las soluciones para que los mismos estudiantes ayuden a sus compañeros a detectar los errores cometidos en la resolución de los problemas planteados por el recurso.

- Idoneidad mediacional, ya que no se precisa de muchos recursos (un solo ordenador en el aula), con lo que los estudiantes pueden jugar colectivamente $y$ se puede trabajar esta situación. La idoneidad aumentaría si se trabaja en el aula de informática donde cada estudiantes o profesor puede explorar el recurso de manera individual o grupal.

- Idoneidad afectiva, que puede ser la más alta de todas ya que este juego propicia el interés y la motivación de los estudiantes, permitiendo un mayor compromiso en su proceso de aprendizaje.

Una selección de juegos que pueden ayudar a los estudiantes se muestra en la tabla 1.

Tabla 1. Juegos relacionados con probabilidad

\begin{tabular}{ll}
\hline \multicolumn{1}{c}{ Nombre } & \\
\hline El circo de la & http://www.harcourtschool.com/activity_es/proba \\
probabilidad & bility_circus \\
\hline El Dado & http://odas.educarchile.cl/objetos_digitales_NE/O \\
& DAS_Matematica/Ed_Matematica/probabilidad_e \\
& vento_experimento_aleatorio/index.html \\
\hline Experiencias & http://www.ceipjuanherreraalcausa.es/Recursosdi \\
Aleatorias & dacticos/SEXTO/datos/03_Mates/datos/05_rdi/ud \\
& 15/1/01.htm \\
\hline Experimental & http://shodor.org/interactivate/activities/ExpProba \\
Probability & bility/ \\
\hline Experimentos & http://www3.gobiernodecanarias.org/medusa/agre \\
Aleatorios & ga/visualizar/es/es- \\
ic_2010051013_9135453/false\#
\end{tabular}

\subsection{Exploración de conceptos}

En esta categoría clasificaremos los recursos encontrados que pueden ser útiles en la visualización 
de diversos objetos matemáticos y que tienen relación con los siguientes temas: azar, tipos de sucesos, experimentos aleatorios y probabilidad.

Ejemplo 2. En la figura 2 mostramos un recurso que aborda algunos objetos matemáticos relacionados con la exploración de los conceptos: probable, seguro e imposible. Estos recursos permiten a los estudiantes visualizar diversos ejemplos de estos conceptos, como también, distinguir entre sus diferencias.

El recurso muestra una serie de ejemplos de situaciones relacionadas con el azar, distinguiendo en sucesos imposible (que un coche funcione sin gasolina), suceso probable (que un perro encuentre un hueso escondido por otro perro) y suceso seguro (sacar un caramelo de menta de una bolsa que solo tiene caramelos de menta). Una vez terminada la animación, se pulsa el botón siguiente, planteándole al usuario una serie de 8 cuestiones de opción múltiple, en donde debe distinguir entre sucesos probables, seguros o imposibles. Una vez elegida la opción, si la respuesta es correcta, se felicita al estudiante y se debe pulsar el botón siguiente para seguir contestando el cuestionario. Si la respuesta no es correcta, se le solicita al estudiante que vuelva a intentarlo.

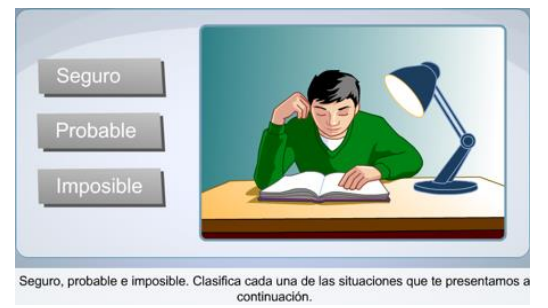

Figura 2. Clasificación de situaciones (seguro, probable, imposible)

http://repositorio.educa.jccm.es/portal/odes/matematicas/a zar_y_probabilidad/mt11_oa01_es/index.html

La principal dificultad que pueden encontrar los estudiantes con este applet es realizar la clasificación de las situaciones, ya que al visualizar algunas de las imágenes relacionadas con los sucesos, estas pueden sugerir una respuesta errónea, dependiendo de la interpretación subjetiva que haga el estudiante de acuerdo a sus concepciones intuitivas del azar.

En este tipo de recursos destaca la idoneidad cognitiva, ya que estos recursos plantean un lenguaje verbal de fácil entendimiento por parte del estudiante, utilizando términos de fácil comprensión y contemplando una serie de representaciones pictóricas que guían al estudiante durante la exploración del recurso. Asimismo, los razonamientos descritos están al alcance de los estudiantes, ya que se ejemplifican situaciones cotidianas relacionadas con las nociones de seguro, probable e imposible.

Una selección de exploradores de conceptos que pueden ayudar a los estudiantes se muestra en la tabla 2. Estos se clasifican en las categorías descritas anteriormente.

Tabla 2. Recursos para la exploración de conceptos

\begin{tabular}{|c|c|c|}
\hline Tipo & Nombre & Dirección \\
\hline \multirow{6}{*}{$\frac{1}{4}$} & $\begin{array}{l}\text { Determinismo y } \\
\text { azar. Baraja }\end{array}$ & $\begin{array}{l}\text { http://ntic.educacion.es/w3/eos/Mate } \\
\text { rialesEducativos/mem2010/labazar/ }\end{array}$ \\
\hline & $\begin{array}{l}\text { Determinismo y } \\
\text { azar. Casilleros } \\
\text { numéricos }\end{array}$ & $\begin{array}{l}\text { http://ntic.educacion.es/w3/eos/Mate } \\
\text { rialesEducativos/mem2010/labazar/ }\end{array}$ \\
\hline & $\begin{array}{l}\text { El azar en la vida } \\
\text { cotidiana }\end{array}$ & $\begin{array}{l}\text { http://repositorio.educa.jccm.es/port } \\
\text { al/odes/matematicas/azar_y_probabi } \\
\text { lidad/mt11_oa02_es/index.html }\end{array}$ \\
\hline & $\begin{array}{l}\text { Experimentos } \\
\text { aleatorios o no } \\
\text { aleatorios }\end{array}$ & $\begin{array}{l}\text { http://contenidos.proyectoagrega.es/ } \\
\text { visualizador- } \\
\text { 1/Visualizar/Visualizar.do?idioma=e } \\
\text { s\&identificador=es_2009091713_88 } \\
\text { 98177\&secuencia=false\# }\end{array}$ \\
\hline & $\begin{array}{l}\text { Los juegos de } \\
\text { azar }\end{array}$ & $\begin{array}{l}\text { http://repositorio.educa.jccm.es/port } \\
\text { al/odes/matematicas/azar_y_probabi } \\
\text { lidad/mt11_oa04_es/index.html }\end{array}$ \\
\hline & Ruleta & $\begin{array}{l}\text { http://nlvm.usu.edu/es/nav/frames_a } \\
\text { sid_186_g_3_t_5.html }\end{array}$ \\
\hline \multirow{8}{*}{ 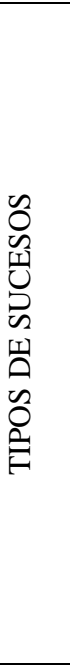 } & $\begin{array}{l}\text { Clasificación de } \\
\text { probabilidades } \\
(\text { actividad } 2)\end{array}$ & $\begin{array}{l}\text { http://repositorio.educa.jccm.es/port } \\
\text { al/odes/matematicas/azar_y_probabi } \\
\text { lidad/mt11_oa01_es/index.html }\end{array}$ \\
\hline & $\begin{array}{l}\text { Determinismo y } \\
\text { azar. ¿Seguro? } \\
\text { ¿Imposible? }\end{array}$ & $\begin{array}{l}\text { http://ntic.educacion.es/w3/eos/Mate } \\
\text { rialesEducativos/mem2010/labazar/ }\end{array}$ \\
\hline & $\begin{array}{l}\text { Experimentos } \\
\text { aleatorios }\end{array}$ & $\begin{array}{l}\text { http://www.librosvivos.net/smtc/ho } \\
\text { metc.asp?temaclave }=1170\end{array}$ \\
\hline & $\begin{array}{l}\text { Random birthday } \\
\text { applet }\end{array}$ & $\begin{array}{l}\text { http://www- } \\
\text { stat.stanford.edu/ susan/surprise/Bir } \\
\text { thday.html }\end{array}$ \\
\hline & $\begin{array}{l}\text { Coin sample } \\
\text { experiment }\end{array}$ & $\begin{array}{l}\text { http://www.math.uah.edu/stat/Applet } \\
\text { s/CoinSampleExperiment.html }\end{array}$ \\
\hline & Dice experiment & $\begin{array}{l}\text { http://www.math.uah.edu/stat/Applet } \\
\text { s/DiceExperiment.html }\end{array}$ \\
\hline & Dice roller & http://www.random.org/dice/ \\
\hline & $\begin{array}{l}\text { Venn diagram } \\
\text { applet }\end{array}$ & $\begin{array}{l}\text { http://www.math.uah.edu/stat/Applet } \\
\text { s/VennGame.html }\end{array}$ \\
\hline \multirow{7}{*}{ 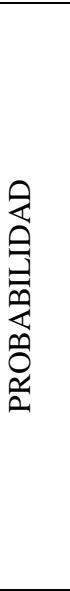 } & $\begin{array}{l}\text { Azar y } \\
\text { probabilidad. al } \\
\text { lanzar un dado }\end{array}$ & $\begin{array}{l}\text { http://ntic.educacion.es/w3/eos/Mate } \\
\text { rialesEducativos/mem2010/labazar }\end{array}$ \\
\hline & $\begin{array}{l}\text { El tablero de } \\
\text { dados }\end{array}$ & $\begin{array}{l}\text { http://www.eduteka.org/MI/master/i } \\
\text { nteractivate/activities/Dice/Index.ht } \\
\text { ml }\end{array}$ \\
\hline & Modelo de cajas & $\begin{array}{l}\text { http://nlvm.usu.edu/es/nav/frames_a } \\
\text { sid_146_g_3_t_5.html }\end{array}$ \\
\hline & $\begin{array}{l}\text { Probabilidad de } \\
\text { un suceso }\end{array}$ & $\begin{array}{l}\text { http://www.librosvivos.net/smtc/ho } \\
\text { metc.asp?temaclave }=1170\end{array}$ \\
\hline & Sacar bola & $\begin{array}{l}\text { http://www.uco.es/ ma1marea/Recu } \\
\text { rsos/Bolas.swf }\end{array}$ \\
\hline & $\begin{array}{l}\text { Situaciones } \\
\text { problemáticas. }\end{array}$ & $\begin{array}{l}\text { http://ntic.educacion.es/w3/eos/Mate } \\
\text { rialesEducativos/mem2010/labazar }\end{array}$ \\
\hline & Tirar el dado & $\begin{array}{l}\text { http://www.uco.es/ ma1marea/Recu } \\
\text { rsos/Dados.swf }\end{array}$ \\
\hline
\end{tabular}




\subsection{Recursos para resolver problemas}

En este apartado analizamos algunos recursos que pueden ayudar al alumno en su resolución de problemas de probabilidad.

Ejemplo 3. El recurso (figura 3) presenta una serie de problemas relativos al cálculo de probabilidad. Se compone de cuatro ventanas despegables, en donde se puede elegir la cantidad de preguntas (largo), el nivel de dificultad (de 1 a 5 , en donde el nivel 1 corresponde al cálculo de probabilidades de experimentos sencillos), la duración (seleccionar el tiempo máximo para contestar las preguntas) y si se necesita pausa entre preguntas. Muestra tres cuadros: las preguntas acertadas (respuestas correctas), las equivocadas (respuestas incorrectas) y el reloj con el tiempo que emplea el usuario para contestar el listado de problemas propuestos.

Una vez contestadas las series de preguntas, el applet entrega un cuadro resumen con:

- Nota (expresada en porcentaje).

- Cumplido (cantidad de respuestas contestadas).

- Sin cumplir (cantidad de respuestas no contestadas).

- Acertado (número de respuestas correctas).

- Equivocado (número de respuestas incorrectas).

- Tiempo (promedio de segundos empleados en contestar una pregunta)

En caso de no contestar correctamente, el resumen también muestra una corrección de los problemas, apareciendo el enunciado de dicho problema, la solución correcta y la respuesta entregada por el usuario. El recurso también entrega un enlace con la posibilidad de que el usuario corrija sus errores, contestando nuevamente los problemas en los que se equivocó al responder el cuestionario.
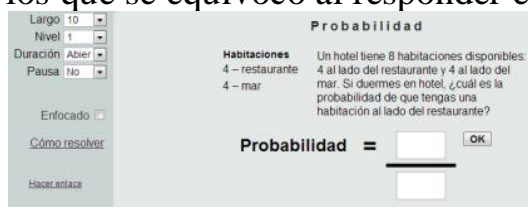

Figura 3. Cuestionario sobre probabilidad

http://www.thatquiz.org/es-d/

Una de las principales dificultades que pueden encontrar los estudiantes tiene relación con el cálculo de probabilidades, ya que pueden confundir la probabilidad simple con la probabilidad de un suceso elemental, confundir la probabilidad con los casos posibles, o calcular la probabilidad de un suceso en vez de la probabilidad de su complementario. Otra dificultad se presenta al anotar el resultado de la probabilidad, pues no se especifica en las instrucciones si se debe o no simplificar.

En este tipo de recursos destaca la idoneidad epistémica o matemática, ya que el número de elementos matemáticos que se describen es alto: Regla de Laplace, cálculo de probabilidades, comparación de probabilidades, etc.

Una selección de recursos para resolver problemas que pueden ayudar a los estudiantes se muestra en la tabla 3.

Tabla 3. Recursos sobre problemas de probabilidad

\begin{tabular}{cc}
\hline Nombre & Dirección \\
\hline $\begin{array}{c}\text { Experiencias } \\
\text { aleatorias }\end{array}$ & $\begin{array}{c}\text { http://www.ceipjuanherreraalcausa.es/Recursos } \\
\text { didacticos/CUARTO/datos/01_Mates/datos/05 } \\
\text { rdi/U15/01.htm }\end{array}$ \\
$\begin{array}{c}\text { Experiencias } \\
\text { aleatorias }\end{array}$ & $\begin{array}{c}\text { http://www.ceipjuanherreraalcausa.es/Recursos } \\
\text { didacticos/CUARTO/datos/01_Mates/datos/05 } \\
\text { rdi/U15/01.htm }\end{array}$ \\
La baraja & http://repositorio.educa.jccm.es/portal/odes/ma \\
española & tematicas/azar_y_probabilidad/mt11_oa03_es/i \\
(actividad & ndex.html \\
N 3) & \\
Probabilidad & http://www.ceipjuanherreraalcausa.es/Recursos \\
de un & didacticos/CUARTO/datos/01_Mates/datos/05 \\
Suceso & rdi/U15/03.htm \\
Probability & http://www.adaptedmind.com/p.php?tagId=10 \\
12 & 12 \\
Problemas y & http://repositorio.educa.jccm.es/portal/odes/ma \\
estrategias & tematicas/azar_y_probabilidad/mt11_oa05_es/i \\
(actividad 1- & ndex.html \\
3) & http://www.ceipjuanherreraalcausa.es/Recursos \\
Sucesos & didacticos/CUARTO/datos/01_Mates/datos/05 \\
posibles, & rdi/U15/02.htm \\
seguros e & \\
imposibles &
\end{tabular}

\subsection{Vídeo tutoriales}

En esta categoría se analizarán y clasificarán una serie de vídeos que muestran una introducción al concepto de probabilidad y que son útiles como apoyo a la enseñanza en educación primaria.

Ejemplo 4. En la figura 5 se muestra una captura del vídeo "Cómo se resuelve un problema de probabilidad", que a partir de una situación cotidiana introduce el concepto de probabilidad y muestra su aplicación a la resolución de problemas. Se analizarán los primeros 03:21 minutos, ya que corresponden al concepto de probabilidad simple. 


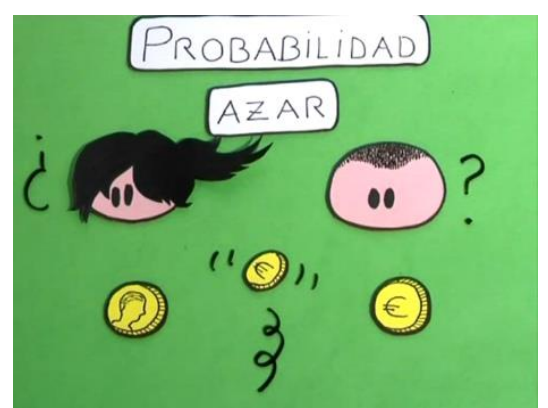

Figura 4. Cómo se resuelve un problema de probabilidad

http://educacion.practicopedia.lainformacion.com/matema ticas/como-se-resuelve-un-problema-de-probabilidad10823

El vídeo plantea una situación cotidiana en donde dos hermanos, María y Jesús, dejan su suerte al azar al intentar decidir quién lavará los platos de la comida, lanzando una moneda al aire. A partir de este ejemplo, se introduce la definición clásica de probabilidad, utilizando Regla de Laplace. El vídeo muestra un ejemplo considerando el suceso: sacar cara al lanzar una moneda. Se complementa la definición dando algunas propiedades de la probabilidad. El vídeo explica que existen distintos problemas de probabilidad y que estos dependen de las condiciones del experimento aleatorio o de las características del suceso. Para ello se ejemplifica con el experimento extracción de bolas negras y rojas de una bolsa, con o sin reposición. Finalmente, se plantea un problema de sucesos, realizando el experimento aleatorio: lanzamiento de un dado, el cual se explica paso a paso, empezando con anotar los datos que nos entrega el enunciado del problema, identificando el espacio muestral y los sucesos "salir par" y "salir primo", que se escriben por extensión, utilizando notación conjuntista, como también su representación gráfica mediante Diagrama de Venn. Posteriormente, se calcula el suceso "Salir Par o Primo" y el suceso "salir Par y Primo", para ambos sucesos, se escriben sus elementos por extensión y se muestra su representación gráfica en Diagrama de Venn.

Una de las dificultades que pueden presentar los estudiantes a la hora de utilizar este recurso en el aula es la poca percepción de los contenidos debido a la velocidad a la que van apareciendo en el vídeo. Es por esta razón que puede servir de complemento al aula, ya que el estudiante puede visualizar el vídeo las veces que necesite.

En este apartado, vuelve a tener gran importancia la idoneidad afectiva, ya que el vídeo propicia el interés y motivación de los estudiantes, permitiendo un mayor compromiso en su proceso de aprendizaje.
Una selección de vídeo-tutoriales que pueden ayudar a los estudiantes se muestra en la tabla 4.

Tabla 4. Videos tutoriales de probabilidad

\begin{tabular}{|c|c|}
\hline Nombre & Dirección \\
\hline $\begin{array}{l}\text { Anticipación de los } \\
\text { resultados de una } \\
\text { experiencia aleatoria }\end{array}$ & $\begin{array}{l}\text { http://www.youtube.com/watch?v=zi } \\
\text { Ng-7p0tWA }\end{array}$ \\
\hline $\begin{array}{l}\text { Conceptos } \\
\text { probabilidad }\end{array}$ & $\begin{array}{l}\text { http://www.youtube.com/watch?v=ka } \\
\text { 53XNic-ew }\end{array}$ \\
\hline $\begin{array}{l}\text { El azar en las } \\
\text { matemáticas }\end{array}$ & $\begin{array}{l}\text { http://www.youtube.com/watch?v=P } \\
\text { mjKP4Y7Nv0 }\end{array}$ \\
\hline $\begin{array}{ll}\begin{array}{l}\text { Escala } \\
\text { probabilidad }\end{array} & \text { de } \\
\end{array}$ & $\begin{array}{l}\text { http://www.youtube.com/watch?v=SZ } \\
\text { eBNolP9gs }\end{array}$ \\
\hline Espacio muestral & $\begin{array}{l}\text { http://www.youtube.com/watch?v=Bt } \\
\text { 711CMenr8 }\end{array}$ \\
\hline $\begin{array}{l}\text { Estadística } \\
\text { probabilidad }\end{array}$ & $\begin{array}{l}\text { http://www.youtube.com/watch?v=4A } \\
\text { nRMRFsk3g\&feature=player_embedd } \\
\text { ed }\end{array}$ \\
\hline $\begin{array}{l}\text { Eventos mutuamente } \\
\text { excluyentes }\end{array}$ & $\begin{array}{l}\text { http://www.youtube.com/watch?v=sd } \\
\text { 133RIDLBA }\end{array}$ \\
\hline $\begin{array}{l}\text { Eventos probables y } \\
\text { poco probables }\end{array}$ & $\begin{array}{l}\text { http://www.youtube.com/watch?v=SX } \\
\text { iL8mgw9fc }\end{array}$ \\
\hline $\begin{array}{l}\text { Identificación de } \\
\text { eventos } \\
\text { complementarios }\end{array}$ & $\begin{array}{l}\text { http://www.youtube.com/watch?v=Sc } \\
\text { 7Iucx6rgc }\end{array}$ \\
\hline $\begin{array}{l}\text { Nociones } \\
\text { probabilidad }\end{array}$ & $\begin{array}{l}\text { http://www.youtube.com/watch?v=YF } \\
\text { EnLoMlsH8 }\end{array}$ \\
\hline Probabilidad & $\begin{array}{l}\text { http://www.youtube.com/watch?v=G } \\
\text { wjbadd3W9Q }\end{array}$ \\
\hline $\begin{array}{l}\text { Probabilidad } 1000 \\
\text { bolas de colores }\end{array}$ & $\begin{array}{l}\text { http://www.youtube.com/watch?v=9s } \\
\text { DItOnHlBk }\end{array}$ \\
\hline Probabilidad básica & $\begin{array}{l}\text { http://www.youtube.com/watch?v=3X } \\
\text { 7t-Q_xejM }\end{array}$ \\
\hline Probabilidad clásica & $\begin{array}{l}\text { http://www.youtube.com/watch?v=kJ } \\
\text { h9z-RdAm4 }\end{array}$ \\
\hline $\begin{array}{l}\text { Probabilidad de un } \\
\text { evento }\end{array}$ & $\begin{array}{l}\text { http://www.youtube.com/watch?v=X } \\
\text { RgvpBlA92U }\end{array}$ \\
\hline $\begin{array}{l}\text { Probabilidad de un } \\
\text { evento simple }\end{array}$ & $\begin{array}{l}\text { http://www.youtube.com/watch?v=7x } \\
\text { Z_kKMiqGU }\end{array}$ \\
\hline $\begin{array}{l}\text { Probabilidades } \\
\text { ejercicios }\end{array}$ & $\begin{array}{l}\text { http://www.youtube.com/watch?v=eX } \\
\text { vP8aKzXDA\&feature=player_embed } \\
\text { ded }\end{array}$ \\
\hline $\begin{array}{l}\text { ¿Qué es más } \\
\text { probable? }\end{array}$ & $\begin{array}{l}\text { http://www.youtube.com/watch?v=kN } \\
\text { UvZGyQOAY }\end{array}$ \\
\hline $\begin{array}{l}\text { Relación entre } \\
\text { probabilidad clásica y } \\
\text { frecuencial }\end{array}$ & $\begin{array}{l}\text { http://www.youtube.com/watch?v=e5 } \\
\text { LghdtWg1U }\end{array}$ \\
\hline Seguro - Imposible & $\begin{array}{l}\text { http://www.youtube.com/watch?v=5T } \\
\text { Nxdd2yAvM }\end{array}$ \\
\hline
\end{tabular}

\section{CONCLUSIONES}

La disponibilidad de recursos libremente accesibles en Internet hace que la cultura y la ciencia se estén democratizando cada vez más, por lo que el aprendizaje se lleva a cabo no solo en el aula tradicional (Galmacci, 2001). El uso de este tipo de recursos aumenta la motivación de los estudiantes por el tema, ya que se presentan los conceptos de una forma más llamativa y permite al estudiante adoptar un papel activo en su aprendizaje. Es por 
ello importante que el profesor tenga en cuenta estos recursos y los incorpore a su enseñanza.

El objetivo principal de este trabajo era encontrar recursos en Internet que pudiesen ayudar a la comprensión de la probabilidad. Hemos elaborado un índice de recursos que abordan los contenidos establecidos en las directrices curriculares, además proporcionan ayudas de aprendizaje, proponen diferentes enfoques para abordar el término probabilidad, plantean problemas relacionados con aspectos de la vida cotidiana, permiten la simulación y experimentación y proporcionan al estudiante un aspecto visual del que carecen los libros de texto. Creemos que la incorporación de estos recursos en el proceso de enseñanza y aprendizaje debidamente planificado y dirigido ayudará al estudiante a comprender las propiedades y aplicaciones de la probabilidad.

Consideramos que todos los recursos analizados presentan una alta idoneidad afectiva, ya que el uso de este tipo de materiales favorece la motivación de los estudiantes por el tema, pues los conceptos se presentan de una forma más atractiva y permite al estudiante adoptar un rol más activo en su proceso de aprendizaje.

Las dificultades encontradas principalmente tienen relación con la interpretación del lenguaje del applet, pues en algunos casos no aparecen todas las instrucciones claramente especificadas. Es en este punto donde el rol docente adquiere importancia como mediador y guía del proceso de aprendizaje de los estudiantes.

Pensamos que un uso adecuado de estas ayudas, debidamente insertado en el proceso de aprendizaje, ayudará al estudiante $\mathrm{o}$ al futuro profesor a comprender las propiedades y aplicaciones de la probabilidad condicional.

\section{REFERENCIAS}

Barragués, J. y Guisasola, J (2007). Simulación por ordenador de experimentos aleatorios en la enseñanza de la probabilidad. SIGMA, 31, 207-223.

Batanero, C., Burrill, G., y Reading, C. (2011). Teaching statistics in school mathematics-Challenges for teaching and teacher education. Netherlands: Springer.

Batanero, C., Fernándes, J. A. y Contreras, J. M. (2009). Un análisis semiótico del problema de Monty Hall e implicaciones didácticas. SUMA, 62, 11-18.

Burrill, G. (2006). NCTM 2006 Yearbook: Thinking and reasoning with data and chance. Reston, VA: NCTM.

Consejería de Educación (2015) Orden de 17 de marzo de 2015, por la que se desarrolla el currículo correspondiente a la Educación Primaria en Andalucía.

Contreras, J. M. (2009). Recursos en Internet para la enseñanza de la probabilidad condicionada. Trabajo de Investigación Tutelada. Universidad de Granada.
Contreras, J. M. (2011). Evaluación de conocimientos y recursos didácticos en la formación de profesores sobre probabilidad condicional. Tesis Doctoral. Universidad de Granada.

Contreras, J. M., Díaz, C., Arteaga, P., Gonzato, M., Cañadas, G. (2011). Probabilidad condicional: Exploración y visualización mediante recursos en Internet. Epsilon. 79, 91-100.

Falk, R., y Konold, C. (1992). The psychology of learning probability. En F. Gordon y S. Gordon (Eds.), Statistics for the twenty-first century (pp. 151-164). Washington, DC: Mathematical Association of America.

Galmacci, G. (2001). The impact of the internet on researchers' training. En Batanero (Ed.), Training researchers in the use of statistics (pp. 159-169). Granada: International Statistical Institute.

Godino, J. D., Batanero, C., y Flores, P. (1999). El análisis didáctico del contenido matemático como recurso en la formación de profesores de matemáticas. En Homenaje al Profesor Oscar Sáenz Barrio (pp. 165-185). Granada: Departamento de Didáctica y Organización Escolar.

Godino, J. D. Batanero, C. y Font, V. (2007). The onto-semiotic approach to research in mathematics education. ZDM. The International Journal on Mathematics Education, 39(1-2), 127-135.

Godino, J. D., Contreras, A. y Font, V. (2006). Análisis de procesos de instrucción basado en el enfoque ontológico-semiótico de la cognición matemática. Recherches en Didactiques des Mathematiques, 26(1), 39-88.

Godino, J. D., Font, V. y Wilhelmi, M. R. (2008). Análisis didáctico de procesos de estudio matemático basado en el enfoque ontosemiótico. Publicaciones, 38, 25-48.

Godino, J., Wilhelmi, M. y Bencomo, D. (2005). Suitability criteria of a mathematical instruction process. A teaching experience of the function notion. Mediterranean Journal for Research in Mathematics Education, 4(2), 1-26.

Godino, J. D., Recio, A. M., Guzmán, R. R., López, F. R., y Pérez, J. L. (2006). Criterios de diseño y evaluación de situaciones didácticas basadas en el uso de medios informáticos para el estudio de las matemáticas. Números, 64, 14-11.

Gómez, E., y Contreras, J. M. (2013). Significados de la probabilidad en el currículo español para la educación primaria. Actas de las I Jornadas Virtuales de Didáctica de la Estadística, Probabilidad y Combinatoria (pp. 571-578). Granada: SEIEM.

Gómez, E. y Contreras, J. M. (2014). Meanings of probability in Spanish curriculum th for primary school». En Proceedings of 9 Statistics. Flagstaff, Arizona: IASE.

Herrera, M. I. y Rodríguez, M. I. (2011). Educación estadística: desarrollo del pensamiento y razonamiento estadístico. XIII CIAEM-IACME, Recife, Brasil.

Jones, G.A., Langrall, C.W. y Mooney, E.S. (2007). Research in probability: Responding to classroom realties. En F. K Lester, Jr. (Ed), Second handbook of research on mathematics teaching and learning (pp. 909-955). New York: Macmillan.

MEC (2014). Real Decreto 126/2014, de 28 de febrero, por el que se establece el currículo básico de la Educación Primaria.

Merriam, S. B., Caffarella, R. S., y Baumgartner, L. M. (2007). Learning in adulthood: A comprehensive guide. San Francisco: John Wiley \& Sons. 
Mills, J. D. (2004). Learning abstract statistics concepts using simulation. Educational Research Quarterly, 28(4), 1833.

Nóbile, C., y Luna, A. E. (2015). Los Entornos Virtuales de Enseñanza y Aprendizaje en la Universidad Nacional de La Plata. Una aproximación a los usos y opiniones de los estudiantes. Innoeduca: international journal of technology and educational innovation, 1(1), 3-9.

Osorio, M. A, Suárez, A. y Uribe, C. (2013). Revisión de alternativas propuestas para mejorar el aprendizaje de la Probabilidad. Revista Virtual Universidad Católica del Norte, 38, 127-142.

Pérez, R., Maya, N., Inzunza, S., Escobar, A., Rosete M. y Romero, M. (2012). Applets interactivos para el aprendizaje de estadística. Universo de la Tecnología, Universidad Tecnológica de Nayarit, 13, 14-17.

Ruiz, K. (2013) Análisis de recursos en internet para la enseñanza de la probabilidad en la educación primaria. Trabajo fin de máster, Universidad de Granada.

Sada, M. (2011). Los applets para la enseñanza de la estadística y probabilidad. Uno Revista de Didáctica de las Matemáticas, 58, 38-48. 